\title{
A Highly-Efficiency NIR Plasmonic Long-Wavelength Cut-Off Filter based on Stepped Impedance Resonators
}

\author{
Seyed Morteza Ebadi ${ }^{1}$, Jonas Örtegren ${ }^{1}$, and Max Yan $^{2}$ \\ ${ }^{1}$ Department of Natural Sciences, Mid Sweden University, SE-851 70 Sundsvall, Sweden \\ ${ }^{2}$ Department of Applied Physics, School of Engineering Sciences, KTH Royal Institute of Technology, SE-11419 Stockholm, Sweden \\ seyed-morteza.ebadi@miun.se
}

\begin{abstract}
We report design and simulation results of a high-efficiency long-wavelength cut-off filter realized by stepped impedance resonators. Moreover, numerical results confirm by modulating the length of resonator, cut-off wavelength can be easily tuned.
\end{abstract}

\section{Introduction}

Surface plasmon polaritons (SPPs), are surface electromagnetic waves that emerge at a metal-insulator interface at high frequencies. Plasmonics are considered to play a significantly important role in development of high-density photonics integrated circuits (PICs), because of its ability to confine light at subwavelength scale and to reduce the size of optical components. SPPs may further work as a platform where photonics and electronic circuits can be mounted on the same chip, which leads to the emergence of various applications [1-3]. There are two types of SPPs-based waveguides: insulator-metal-insulator (IMI), and metal-insulator-metal (MIM). The MIM configuration is of great interest due to its strong mode confinement, while losses in the waveguide can be assumed to be at an acceptable level. There are miscellaneous conceivable applications based on the MIM waveguide structure including sensors [4], modulators [5], and so forth have been suggested. Examples include optical filters with widespread applications in wavelength division multiplexing (WDM) in optical communication systems, dispersion compensation by means of minimizing the chromatic dispersion, reduction of luminous systems, and remote sensing methods such as light detection and ranging (LIDAR). Consequently, we have designed and proposed a highly efficient long-wavelength cut-off filter based on stepped impedance resonators (SIRs). The new symmetric nanoplasmonic filter is extremely compact and functions at near-infrared (NIR) wavelengths with high-efficiency. The ability to tune the optical output signal is achieved by controlling the length of the step discontinuity. It is noteworthy to point out that the structural parameters are optimized against the size and efficiency, as well as to provide ease of fabrication due to its symmetric configuration.

\section{Device Configuration and Numerical Results}

Figure 1. (a) schematically displays the configuration of the suggested novel nanoplasmonic long-wavelength cut-off filter.
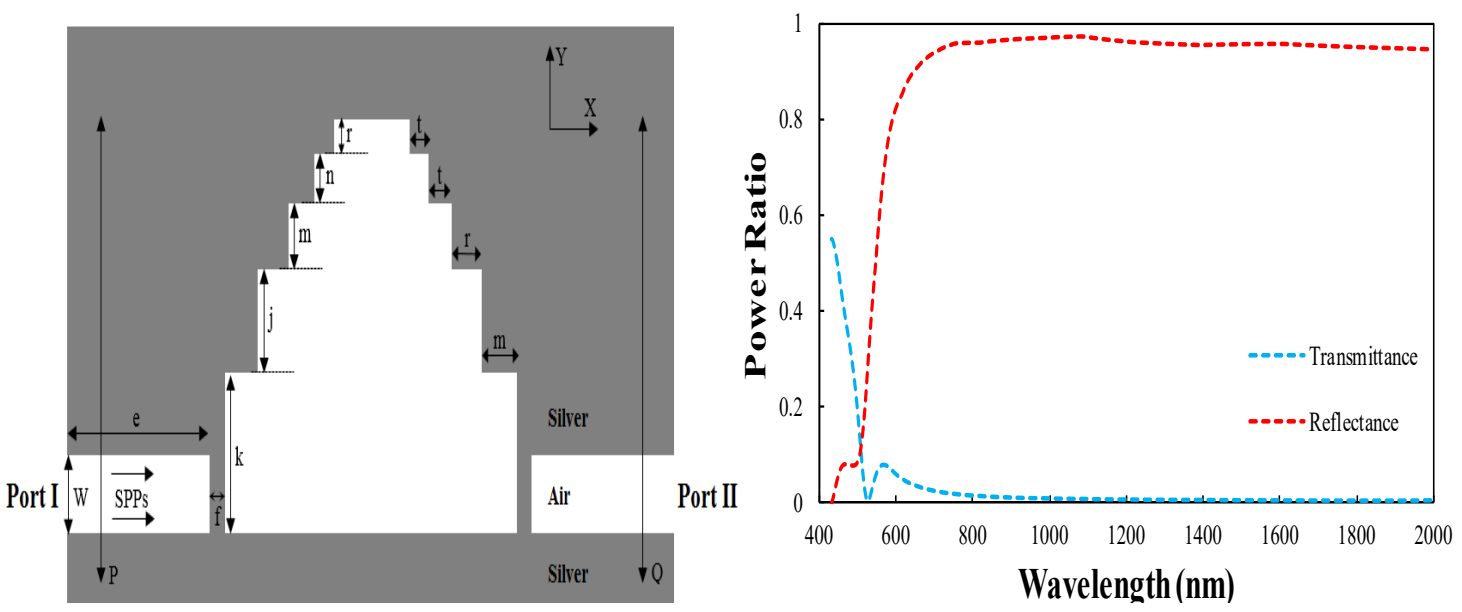

Fig. 1. (a) Schematic configuration of proposed band-stop plasmonic filter. (b) Transmission spectra of the band-stop wavelength filter for W=25 $\mathrm{nm}, \mathrm{e}=50 \mathrm{~nm}, \mathrm{f}=\mathrm{t}=5 \mathrm{~nm}, \mathrm{k}=50 \mathrm{~nm}, \mathrm{j}=30 \mathrm{~nm}, \mathrm{~m}=20 \mathrm{~nm}, \mathrm{n}=15 \mathrm{~nm}$, and $\mathrm{r}=10 \mathrm{~nm}$. 
The suggested device structure is composed of two metallic layers, that are set to be silver with complex dielectric constant taken from experimental data by Johnson and Christy [6], while the insulator medium is set to be air. The following assumption are made to attain the numerical results in this letter; a dipole source is employed to excite the fundamental TM mode of the plasmonic waveguide. The grid size is set to be $6 \mathrm{~nm} \times 6 \mathrm{~nm}$, along the $\mathrm{x}$ and y directions. As illustrated in Fig. 1. (a), two power monitors, P and Q, are located to capture the incident and transmitted power in the structure while the light is launched to the structure from the left port, and the transmittance of the device can therefore be defined as T=Pout/Pi. We have used a full-wave EM tool, CST MWS, whose frequency domain solver is based on the finite element method (FEM), to achieve the numerical results in this letter. Figure 1. (b) demonstrates the numerical results of the miniaturized and tunable cut-off filter from at NIR wavelengths for the physical parameters $\mathrm{W}=25 \mathrm{~nm}$, (the width of the input port), $\mathrm{f}=5 \mathrm{~nm}$, (the coupling distance between the bus waveguide and SIR), $\mathrm{e}=50 \mathrm{~nm}$ (the length of the bus waveguide), and the parameters of the SIR are as follows; $k=50 \mathrm{~nm}, j=30 \mathrm{~nm}, \mathrm{~m}=20 \mathrm{~nm}, \mathrm{n}=15 \mathrm{~nm}$, and $\mathrm{r}=10 \mathrm{~nm}$, the lengths of the first- to the fifth step discontinuity, respectively. The widths of the SIR are shown in Fig. 1. (a) is set to have the following relation, $\mathrm{t}=\mathrm{f}$. The cut-off wavelength is specified as the wavelength where the transmission spectrum is 1\% [7], and is found to be $999 \mathrm{~nm}$. The transmission efficiency remains less than $1 \%$ up to the wavelength of $2 \mu \mathrm{m}$, while the lowest value is found to be $0.05 \%$ at wavelength $1873.7 \mathrm{~nm}$.

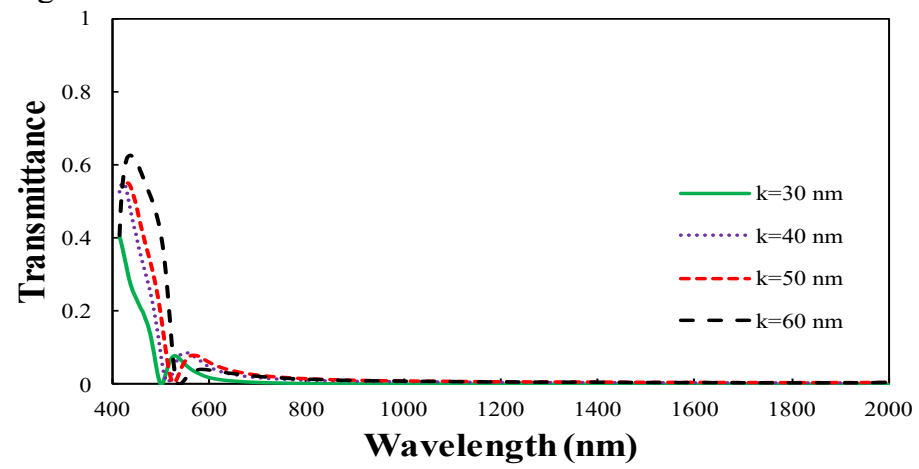

Fig. 2. The transmission profile as a function of the wavelength for the suggested long-wavelength cut-off filter with various lengths of the stepped impedance resonators, $\mathrm{k}$.

Figure 2 demonstrates the simulation results of the transmission spectra of the suggested tunable longwavelength cut-off wavelength filter for $\mathrm{k}=30 \mathrm{~nm}, 40 \mathrm{~nm}, 50 \mathrm{~nm}, 380 \mathrm{~nm}$, and $60 \mathrm{~nm}$, while all other parameters of the device are kept the same as in Fig. 1 (a). The cut-off wavelengths are found to be $657 \mathrm{~nm}, 810 \mathrm{~nm}, 999 \mathrm{~nm}$, and $1060 \mathrm{~nm}$, respectively. It is thus, demonstrated that by tuning the length of SIRs, the cut-off wavelength can be readily modulated, offering an approach to design and control the optical output signal.

\section{Conclusion}

In summary, a novel, highly compact, and tunable NIR long-wavelength cut-off filter based on SIRs is proposed and numerically investigated. Furthermore, compared to recent studies [8-9], not only does the proposed structure provides smaller footprint, but at the same time offers higher efficiency, which may find applications in image processing systems and in high-density PICs.

\section{References}

[1] Gramotnev, Dmitri K., and Sergey I. Bozhevolnyi. "Plasmonics beyond the diffraction limit." Nature photonics 4.2 (2010): 83-91.

[2] Atwater, Harry A. "The promise of plasmonics." Scientific American 296.4 (2007): 56-63.

[3] Ozbay, Ekmel. "Plasmonics: merging photonics and electronics at nanoscale dimensions." science 311.5758 (2006): 189-193.

[4] Al Mahmud, Rabiul, et al."Plasmonic Refractive Index Sensor Based on Ring-Type Pentagonal Resonator with High Sensitivity." Plasmonics 16.3 (2021): 873-880.

[5] Burla, Maurizio, et al. "500 GHz plasmonic Mach-Zehnder modulator enabling sub-THz microwave photonics." APL Photonics 4.5 (2019): 056106.

[6] P. B. Johnson and R.-W. Christy, "Optical constants of the noble metals," Physical review B, vol. 6, no. 12, p. 4370, 1972.

[7] S. M. Ebadi, et al, "A Multipurpose and Highly-Compact Plasmonic Filter Based on Metal-Insulator-Metal Waveguides," IEEE Photonics Journal, vol. 12, no. 3, pp. 1-9, June 2020.

[8] Mishra, Manoj, Mohit Sharma, and Prachi Gupta. "Compact MIM Plasmonic Ring Resonator for Nano-Interconnect Applications." Physica E: Low-dimensional Systems and Nanostructures (2021): 114711.

[9] S. M. Ebadi, and J. Örtegren. "A Highly-Efficient and Compact Surface Plasmon Polaritons High-Pass Filter based on MIM waveguides." Laser Science. Optical Society of America, 2020. 